\title{
VIRULENCE OF BORDETELLA BRONCHISEPTICA FROM PIGS WITH OR WITHOUT ATROPHIC RHINITIS
}

\author{
J. M. Rutter, Lesley M. A. Francis AND \\ B. F. SANSOM \\ Agricultural Research Council, Institute for Research on Animal Diseases, \\ Compton, Newbury, Berkshire
}

\begin{abstract}
SUMmaRy. The virulence of 17 isolates of Bordetella bronchiseptica from 13 pig herds was compared by intranasal infection of gnotobiotic piglets and LD50 tests on mice. Of 59 piglets given $8 \cdot 1-10 \cdot 5 \log _{10}$ colony-forming units (cfu) of isolates from two herds with atrophic rhinitis (AR isolates) or isolates from six unaffected herds (non-AR isolates), 16 died of acute pneumonia; the survivors developed non-progressive turbinate hypoplasia and chronic pneumonia. Infection of 11 piglets with c. 3.0 $\log _{10} \mathrm{cfu}$ of three AR isolates or three non-AR isolates caused turbinate hypoplasia, but only slight pneumonia and no deaths. There were no significant differences between the virulence of $A R$ and non-AR isolates in piglets. In LD50 tests in mice, there were no significant differences between the results from six AR isolates and six non-AR isolates, or from toxin prepared from two AR isolates and one non-AR isolate. It was concluded that the virulence of $A R$ and non-AR isolates was fairly uniform, and that other factors must be responsible for the occurrence of progressive lesions of atrophic rhinitis in some but not all infected herds.
\end{abstract}

\section{INTRODUCTION}

Atrophic rhinitis is an economically important disease of fattening pigs. The signs include shortening or twisting of the snout, hypoplasia or disappearance of the turbinate bones, nasal discharge and haemorrhage, pneumonia, and impaired growth (Switzer and Farrington, 1976). The aetiology has for many years been controversial, but in the USA and Japan Bordetella bronchiseptica is now regarded as the principal cause (Switzer and Farrington, 1976). In England, B. bronchiseptica is present in most pig herds (Cameron, Giles and Smith, 1980; Giles et al., 1980; Rutter, 1981), but some infected herds show either no clinical signs of disease (Alexander et al., 1980) or only minor abnormalities of the turbinate bones (Rutter, 1981). This might be explained by variations in virulence, for which there is some evidence (Ross, Switzer and Duncan, 1967; Miniats and Johnson, 1980; Skelly et al., 1980). However, adequate comparisons between B. bronchiseptica from herds with or without clinical disease have not been made. The purpose of this study was to compare the virulence of such isolates and their ability to colonise the nasal cavity of pigs. 


\section{MATERIALS AND METHODS}

Piglets. Eight-six piglets from nine litters were used (table I). The piglets were derived by hysterotomy and reared in pairs in plastic isolators (Tavernor et al., 1971; Trexler, 1971). Seventy-eight were germ-free and eight were found to be contaminated with a gram-positive coccus before infection, but this did not appear to affect the results. The piglets were fed sterile milk (Carnation) diluted with a solution of mineral supplement. The piglets from litter 8 were weaned on irradiated piglet-grower ration at 6 weeks of age and transferred to isolation boxes at 10 weeks. In experiments 1 and 2 the effects of giving large or small numbers of AR or non-AR isolates were compared in piglets killed 28 days after infection. In experiment 3 , one AR isolate was used; two infected animals and one control were killed at 28 days, three infected and three controls at 63 or 68 days, and one control and two infected at 166 days. Two piglets in litter 8 died of Escherichia coli septicaemia after being transferred to the isolation boxes.

$B$. bronchiseptica isolates. Seventeen isolates were tested in pigs and mice: eight, from seven herds with clinical atrophic rhinitis (AR isolates), comprised four from pigs aged 10-16 weeks with severe disease (nos. 276, MH7, AS4 and GFS3C) and four from unaffected pigs aged 6 weeks (nos. AS2, SW6, GF12, and Y2); nine isolates (nos. IM5, FW5, FW7, LI, OP1, CWT2 and CWT10, CTH5 and CTH19) were from pigs aged 6 weeks in six herds in which clinical atrophic rhinitis had never been observed (non-AR isolates). Isolate 276 had been freeze-dried since 1972; the others were isolated in 1978-79 and freeze-dried immediately. All the isolates produced phase-I colonies on Bordet-Gengou (BG) agar (Nakase, 1957a). Phase-III colonies were produced from phase I by repeated subculture in peptone water (Nakase, 1957b); they differed from phase-I colonies in their quicker growth on blood agar, lack of haemolytic property, and larger, flatter, greyer appearance after incubation for $48 \mathrm{~h}$.

Infection procedures. Confluent growth of $B$. bronchiseptica on horse-blood or BG agar incubated for $24 \mathrm{~h}$ was diluted in phosphate-buffered saline (PBS) to give the numbers $/ \mathrm{ml}$ shown in table I. The suspensions were sealed in sterile glass ampoules for transfer into the isolators. Piglets were infected at 7 days of age by dropping $0.5 \mathrm{ml}$ of bacterial suspension into each nostril. Viable bacteria were counted in the suspensions transferred out of the isolators.

Post-infection procedures. Rectal temperatures (litters 2-4) were taken and the piglets were weighed (litters 1-4) daily. All were observed for clinical signs of infection. Nasal swabs were collected once or twice each week for counting of B. bronchiseptica (Rutter, 1981). Blood was withdrawn each week from the anterior vena cava and mixed with heparin or allowed to coagulate overnight to yield serum.

Haematological and serological techniques. Differential leucocyte counts (litters 2-4) were made on blood smears stained by Leishman's method. The total erythrocyte and leucocyte counts, packed-cell volume (PCV), mean corpuscular volume (MCV), and haemoglobin ( $\mathrm{Hb})$ content of all litters were measured in a Coulter counter (FN6/C, Coulter Electronics, Coldharbour Lane, Harpenden, Herts AL5 4UN). Plasma (litters 2 and 3) was analysed for calcium and magnesium by atomic absorption spectrophotometry in the presence of $1 \%$ lanthanum chloride; for inorganic phosphorus by the method of Robinson, Roughan and Wagstaff (1971); and for alkaline phosphatase (EC 3.1.3.1) by means of the Boehringer Kit 123 889 and a Vitatron Automatic Kinetic Enzyme and Substrate Analyser (MSE Scientific Instruments, Manor Royal, Crawley, Sussex).

Micro-agglutination tests for antibody to $B$. bronchiseptica were done in plastic trays with $0 \cdot 25-\mu \mathrm{l}$ volumes of antigen and serum. The procedure was similar to that of Jenkins (1978); serum was diluted 10-2560 times, and the end point was the last well showing $50 \%$ agglutination.

Necropsy, histology and analytical procedures. Piglets were killed by intravenous injection of pentobarbitone. The snout was sectioned transversely with a saw midway between the canine and first cheek tooth (experiments 1 and 2), or at the first cheek tooth (experiment 3). The size of the turbinates was estimated on a scale (Done, Richardson and Hebert, 1964) from 0 (normal) to grade 5 (complete disappearance of turbinates). Snout sections were fixed in neutral buffered formalin, decalcified in $10 \%$ formic acid and stained with haematoxylin and eosin (HE). The dimensions of the turbinates (area, perimeter, height, and width) were measured by tracing a projected magnified image on a quantitative image analyser (Kontron, MOP/AMO/, British American Optical, 820 Yeovil Road, Slough, Berks). The lungs were removed, and cultured; 
swabs were taken from the pharynx, trachea, spleen, liver, kidney, and heart blood and cultured on horse-blood agar and BG-agar plates.

In litters 2 and 3 , the snout was sectioned through the ethmoturbinates and halved longitudinally through the nasal septum. Sections made from one half were stained with HE; the turbinate and facial bones were separated from the other half and stripped of muscle and connective tissue. The bones were weighed, dried in an oven at $100^{\circ} \mathrm{C}$ overnight, reweighed, and ashed in a muffle furnace at $500^{\circ} \mathrm{C}$ overnight. The ash was weighed and dissolved in $2 \mathrm{~N} \mathrm{HCl}$. The solution was analysed for calcium, phosphorus, and magnesium by the methods used for plasma, and also for sodium, potassium, iron, zinc, and copper by atomic absorption spectrophotometry.

LD50 tests. Bacteria from 24-h cultures on BG agar of AR isolates SW6, GF12, 276, MH7, AS2 and Y2, and non-AR isolates LI, CWT2, CTH5, IM5, FW7 and OP1, were diluted in sterile peptone water to give $c .110 \mathrm{mg} / \mathrm{ml}$. Six dilutions $\left(10^{1}-10^{6}\right)$ were made in peptone water and 0.5 $\mathrm{ml}$ of each was injected intraperitoneally into groups of six white mice, each weighing $c .20 \mathrm{~g}$. Deaths were recorded up to 14 days, and the LD50 of each isolate was calculated (Reed and Muench, 1938). Viable bacteria in the dilutions were counted on BG agar. Toxin was assayed as follows in AR isolates 276 and $\mathrm{MH} 7$, and non-AR isolate IM5. Growth from 10 BG-agar plates was diluted tenfold and then frozen $\left(-70^{\circ} \mathrm{C}\right)$ and thawed $\left(37^{\circ} \mathrm{C}\right) 20$ times. The suspension was centrifuged at $2000 \mathrm{~g}$ for $30 \mathrm{~min}$ at $4^{\circ} \mathrm{C}$, and the supernate filtered through a $0.45-\mu \mathrm{m}$ Millipore filter. After being diluted 5-200 times in saline, it was injected intraperitoneally in $0 \cdot 5-\mathrm{ml}$ volumes into groups of six mice.

Statistical analyses. Rectal temperatures, weights, blood and plasma values, nasal swab counts, and turbinate size were examined by analysis of variance, by the method of general least squares for non-orthogonal data. Because repeated observations were made on blood and plasma values, and nasal swab counts, the "split-plot in time" model (Steel and Torrie, 1960) was used; an example with the degrees of freedom for the analysis of swab counts in experiment 1 is given (table II). When observations were made on each side of the snout (turbinate size, swab counts), a similar subdivision of the analysis of variance was made so that the effects of side and side $\times$ isolate interaction could be treated as "subplot" effects.

Probit analysis was used to compare the LD50s of different isolates.

\section{RESULTS}

\section{Mortality}

Of 59 piglets given $8 \cdot 1-10 \cdot 5 \log _{10} \mathrm{cfu}$ of two AR isolates or 6 non-AR isolates (table I), 16 died of an acute haemorrhagic pneumonia. AR and non-AR isolates caused mortality. Most piglets died within 2 days of infection with firm plum-coloured lesions in the right ventral lobes of the lungs (Meyer and Beamer, 1973) and large quantities of bloodstained serous fluid in the pleural cavities and pericardial sac. The lung lesions were more extensive in piglets that died after 3 days. Profuse pure cultures of $B$. bronchiseptica were isolated from the pneumonic lungs of the dead piglets. In litter 6, 10 of 12 piglets died after being given $0 \cdot 2-0.7 \log _{10}$ cfu more bacteria than had been given to litter 5, in which only 1 of 12 died; however, it was not clear whether the difference was attributable to the greater dose or to litter variation.

\section{Experiment 1: comparison of piglets infected (c. $9 \cdot 8 \log _{10} \mathrm{cfu}$ ) with $A R$ isolates (276 and MH7) and a non-AR isolate (IMS) for 28 days}

Clinical signs. Some piglets coughed or sneezed occasionally and were partially anorexic for a few days. Transient nasal discharges were observed, 
TABLE I

Infection of gnotobiotic piglets with B. bronchiseptica

\begin{tabular}{|c|c|c|c|c|c|c|c|c|c|c|c|c|}
\hline \multirow{3}{*}{$\begin{array}{c}\text { Experi- } \\
\text { ment } \\
\text { no. }\end{array}$} & \multirow{3}{*}{$\begin{array}{c}\text { Litter } \\
\text { no. }\end{array}$} & \multicolumn{11}{|c|}{ Dose ( $\left.\log _{10} \mathrm{cfu}\right)$ of $B$. bronchiseptica given to the stated number of piglets } \\
\hline & & \multirow{2}{*}{$\begin{array}{c}\text { None } \\
\text { (control) }\end{array}$} & \multicolumn{4}{|c|}{$\mathrm{AR}$ isolates } & \multicolumn{6}{|c|}{ Non-AR isolates } \\
\hline & & & 276 & MH7 & AS4 & GFS3C & IM5 & FW5 & LI & OPI & CWT 10 & CTH19 \\
\hline \multirow{5}{*}{1} & 1 & $0(2)$ & $10 \cdot 2(2)$ & $10 \cdot 3(2)$ & $\ldots$ & $\ldots$ & $10 \cdot 0(2)$ & $\ldots$ & $\ldots$ & $\ldots$ & $\ldots$ & $\ldots$ \\
\hline & 2 & $0(4)$ & $10 \cdot 1(5)$ & $\ldots$ & $\ldots$ & $\ldots$ & $\ldots$ & $\ldots$ & $\ldots$ & $\ldots$ & $\ldots$ & $\ldots$ \\
\hline & 3 & $0(3)$ & $9 \cdot 8(2)$ & $9 \cdot 7(4)$ & $\cdots$ & $\cdots$ & $\begin{array}{c}9 \cdot 9(4) \\
{[3]}\end{array}$ & $\cdots$ & $\cdots$ & $\cdots$ & $\ldots$ & $\cdots$ \\
\hline & 4 & $0(1)$ & $\cdots$ & $\begin{array}{c}10 \cdot 3(2) \\
{[1]}\end{array}$ & $\cdots$ & $\ldots$ & $8 \cdot 1(3)$ & $\ldots$ & $\ldots$ & $\ldots$ & $\cdots$ & $\ldots$ \\
\hline & 5 & $0(0)$ & $10 \cdot 0(2)$ & ... & $\ldots$ & $\ldots$ & $\cdots$ & $9 \cdot 8(2)$ & $9 \cdot 7(2)$ & $\begin{array}{c}9 \cdot 9(2) \\
{[1]}\end{array}$ & $9 \cdot 8(2)$ & $9 \cdot 6(2)$ \\
\hline 2 & 6 & $0(0)$ & $\begin{array}{c}10 \cdot 3(2) \\
{[1]}\end{array}$ & $\cdots$ & $\cdots$ & $\cdots$ & $\ldots$ & $\begin{array}{c}10 \cdot 5(2) \\
{[2]}\end{array}$ & $\begin{array}{c}10 \cdot 2(2) \\
{[2]}\end{array}$ & $\begin{array}{c}10 \cdot 1(2) \\
{[2]}\end{array}$ & $\begin{array}{c}10 \cdot 2(2) \\
{[1]}\end{array}$ & $\begin{array}{c}10 \cdot 2(2) \\
{[2]}\end{array}$ \\
\hline \multirow{3}{*}{3} & 7 & $0(0)$ & $3 \cdot 1(1)$ & $\cdots$ & $3 \cdot 0(2)$ & $2 \cdot 9(2)$ & $\ldots$ & .. & $2 \cdot 9(2)$ & .. & $3 \cdot 0(2)$ & $2 \cdot 9(2)$ \\
\hline & 8 & $0(3)$ & $\begin{array}{c}9 \cdot 1(5) \\
{[1]^{*}}\end{array}$ & $\cdots$ & $\cdots$ & $\cdots$ & $\ldots$ & $\ldots$ & $\ldots$ & $\ldots$ & $\cdots$ & $\cdots$ \\
\hline & 9 & $0(3)$ & $8 \cdot 3(4)$ & $\ldots$ & $\ldots$ & $\ldots$ & $\cdots$ & $\cdots$ & $\cdots$ & $\cdots$ & $\cdots$ & $\cdots$ \\
\hline
\end{tabular}

( ) = Number of piglets; [ ] = number that died.

* Two other piglets died (see Methods).

but the weight of nasal swabs from uninfected piglets was $17.0 \mathrm{mg}$ (mean value) compared with $16 \cdot 8-17 \cdot 4 \mathrm{mg}$ for the infected groups; the differences were not significant at any time after infection, or between litters, or between the right and left nasal cavities. Infection did not cause pyrexia or reduce weight gains. A slight deviation of the snout to the right was observed in two piglets infected with AR isolate 276, and to the left in two piglets infected with AR isolate MH7. Subsequent experiments indicated that a slight deviation at 28 days was not progressive.

Colonisation of the nasal cavity. Counts of $B$. bronchiseptica reached $c .8 \cdot 0$ $\log _{10}$ cfu on day 2 after infection and declined to $c .7 \cdot 3 \log _{10}$ cfu by day 28 . The mean count of isolate MH7 $\left(7.72 \log _{10} \mathrm{cfu}\right)$ was greater than that of isolate 276 $\left(7.40 \log _{10}\right.$ cfu; $p<0.05$; average SE of the difference between the means of two isolates $=0.09)$, but neither differed significantly from that of isolate IM5 (7.66 $\log _{10} \mathrm{cfu}$ ). There were no differences between counts from the right and left nasal cavities, or on different days after infection. The statistical analysis is exemplified in table II. Mean counts of the three isolates in litters 1 and 2 (7.76 and $\left.7.80 \log _{10} \mathrm{cfu}\right)$ were 2-3 times greater than those of litters 3 and 4 (7.40 and $7 \cdot 31 \log _{10} \mathrm{cfu} ; \mathrm{p}<0.01$; average SE of the difference between the means of two litters $=0 \cdot 13$ ); this was presumably due to litter variation, but there were no significant differences in the clinical and necropsy findings.

Changes in blood and plasma. There were no differences in the erythrocyte count, $\mathrm{PCV}, \mathrm{MCV}$ or $\mathrm{Hb}(\mathrm{g} / 100 \mathrm{ml})$ of infected and uninfected piglets. In the infected piglets there was a leucocytosis on days 7 and $14(p<0.01)$, but there were no differences between the infected groups (table III). Infected piglets had an increased neutrophil count on days 7 and $14(\mathrm{p}<0.05)$ and a lymphocytosis on days 7, 14 and $21(\mathrm{p}<0.05)$; for isolate 276 , the counts decreased more slowly from day 14-28. There were no changes in the calcium, 
TABLE II

Statistical analysis of swab counts in experiment I

\begin{tabular}{lc}
\hline \multicolumn{1}{c}{ Source } & Degrees of freedom \\
\hline Litter & 3 \\
Isolate & 2 \\
Litter $\times$ isolate & 6 \\
Error & 10 \\
$\quad$ Total (piglets) & 21 \\
Time & 4 \\
Time $\times$ isolate & 8 \\
Error & 76 \\
$\quad$ Total & 109 \\
\end{tabular}

phosphorus or magnesium concentrations, or in alkaline phosphatase activity, in the plasma of infected and uninfected piglets.

Agglutinating antibodies to B. bronchiseptica in serum were detected neither before infection nor in uninfected piglets. In infected animals antibodies were detected on day 7 , and by day 14 the mean titres were 1814 (non-AR isolate IM5), 2109 (AR isolate MH7), and 1011 (AR isolate 276); the differences were not significant and the titres were maintained until the piglets were killed.

Necropsy observations. The turbinate bones of the infected piglets showed grade- 3 lesions. This was confirmed by weighing the ventral turbinates and by measurements of sections stained with HE. The wet weight of the turbinates of infected piglets was $30-55 \%$ less than that of the controls, and there were similar decreases in dry weight and ash content. However, there was no change in the proportions of calcium, phosphorus, magnesium, sodium, potassium, iron, zinc, and copper in the ash. There were no significant differences between infected and control piglets in the wet weights, dry weights, ash weights, and composition of the facial bones.

$\mathrm{HE}$-stained sections of the ventral turbinate bones were smaller in infected

TABLE III

Experiment 1: mean leucocyte counts in litters 2-4

\begin{tabular}{|c|c|c|c|c|c|}
\hline \multirow{2}{*}{$\begin{array}{l}\text { Days after } \\
\text { infection }\end{array}$} & \multicolumn{4}{|c|}{$\begin{array}{l}\text { Mean number of leucocytes }\left(10^{3} / \mathrm{mm}^{3}\right) \\
\text { in piglets infected with isolate }\end{array}$} & \multirow{2}{*}{$\begin{array}{c}\text { Average SE of } \\
\text { the difference } \\
\text { between two } \\
\text { isolate means }\end{array}$} \\
\hline & $\begin{array}{c}\text { None } \\
\text { (control) }\end{array}$ & 276 & $\mathrm{MH} 7$ & IM5 & \\
\hline 0 & $\begin{array}{l}5 \cdot 8 \\
(8)\end{array}$ & $\begin{array}{l}7 \cdot 0 \\
(7)\end{array}$ & $\begin{array}{l}6 \cdot 9 \\
(5)\end{array}$ & $\begin{array}{l}6 \cdot 0 \\
(4)\end{array}$ & $3 \cdot 26$ \\
\hline 7 & $7 \cdot 1$ & $19 \cdot 1$ & $20 \cdot 5$ & 18.5 & $3 \cdot 21$ \\
\hline 14 & 8.9 & $15 \cdot 3$ & $17 \cdot 7$ & $17 \cdot 0$ & $3 \cdot 21$ \\
\hline 21 & $7 \cdot 4$ & $13 \cdot 1$ & $11 \cdot 1$ & $12 \cdot 7$ & $3 \cdot 25$ \\
\hline 28 & $6 \cdot 2$ & $9 \cdot 3$ & 4.6 & $4 \cdot 1$ & $3 \cdot 74$ \\
\hline $\begin{array}{l}\text { Average SE } \\
\text { of the } \\
\text { difference } \\
\text { between two } \\
\text { times }\end{array}$ & $1 \cdot 89$ & 1.95 & $2 \cdot 44$ & 2.63 & \\
\hline
\end{tabular}

()$=$ Number of piglets. 
piglets ( $\mathrm{p}<0.001$, table IV) but the differences between infected groups were not significant except that the turbinate width was larger in pigs infected with non-AR isolate IM5 $(\mathrm{p}<0.05)$.

Deviation of the nasal septum occurred in one of 10 controls and eight of 22 infected piglets. The deviation was to the left in all except one piglet and occurred most frequently and severely in piglets infected with AR isolate 276.

All 22 infected piglets had fibrosing pulmonary lesions (Duncan, Ramsey and Switzer, 1966). The right cardiac lobe was affected in 21 pigs, the right apical in 16, left apical in 14, right diaphragmatic in nine, intermediate in six, and left diaphragmatic in four. The percentage consolidation of individual lobes was also assessed for the three isolates, but there were no significant differences.

Profuse pure cultures of $B$. bronchiseptica were grown from the pneumonic lungs and trachea of the infected piglets, but fewer organisms were recovered

TABLE IV

Experiment 1: size of the ventral turbinate bones in litters 1-4

\begin{tabular}{l|cccc}
\hline \multirow{2}{*}{$\begin{array}{l}\text { Piglets infected } \\
\text { with isolate }\end{array}$} & \multicolumn{4}{|c}{$\begin{array}{c}\text { Mean dimensions of the ventral turbinate bones } \\
\text { (adjusted for litter varation) }\end{array}$} \\
\cline { 2 - 5 } & $\begin{array}{c}\text { Area } \\
\left(\mathrm{mm}^{2}\right)\end{array}$ & $\begin{array}{c}\text { Perimeter } \\
(\mathrm{mm})\end{array}$ & $\begin{array}{c}\text { Height } \\
\text { (mm) }\end{array}$ & $\begin{array}{c}\text { Width } \\
\text { (mm) }\end{array}$ \\
\hline None (control) & $\begin{array}{r}20.1 \\
(9)\end{array}$ & 37.2 & 9.7 & 4.4 \\
276 & $\begin{array}{r}7.5 \\
(9)\end{array}$ & 18.0 & 5.4 & 2.6 \\
MH7 & $\begin{aligned} 6.8 \\
(7)\end{aligned}$ & 19.5 & 5.9 & 2.7 \\
IM5 & $\begin{aligned} 9.7 \\
(6)\end{aligned}$ & 21.4 & 5.9 & 3.6 \\
& & & & \\
$\begin{array}{l}\text { Average SE of } \\
\text { difference between two } \\
\text { isolate means }\end{array}$ & 1.55 & 2.91 & 0.59 & 0.29 \\
\hline
\end{tabular}

()$=$ Number of sections; one control section was incomplete and omitted

from the pharynx. The organism was also cultured in small numbers, generally $<10$ colonies/swab, from 36 of 88 swabs taken at necropsy from the heart blood, liver, spleen, and kidneys.

Histopathological changes. Changes in the ciliated columnar epithelium and lamina propria similar to those described by Duncan et al. (1966) were observed in sections of the ventral turbinate bone. In addition, 20 of 22 infected pigs had areas of cartilage in the bony core of the turbinates; these varied from small islands at the tips of the scrolls to areas occupying almost one-third of the bony core. Small areas of cartilage were seen in only three of 10 control animals. There were no obvious differences between the histopathological lesions produced by the three isolates. 
Experiment 2: comparison of piglets infected (c. 9.8 or $3.0 \mathrm{cfu}$ ) with three $A R$ and five non- $A R$ isolates

Of 12 piglets in litter 6 , ten died after infection and the results from this litter were omitted. All eight isolates (AR isolates 276, AS4, GFS3C; non-AR isolates FW5, LI, OP1, CWT10, CTH19) colonised the nasal cavity, the only difference being that peak bacterial counts were attained 4 days later in pigs given the lower dose (fig. 1). There was a leucocytosis from days 9-21 $\left(25 \cdot 2-16.5 \times 10^{3} / \mathrm{mm}^{3} ; \mathrm{p}<0.05\right)$ in pigs given the higher dose, and at 9 days

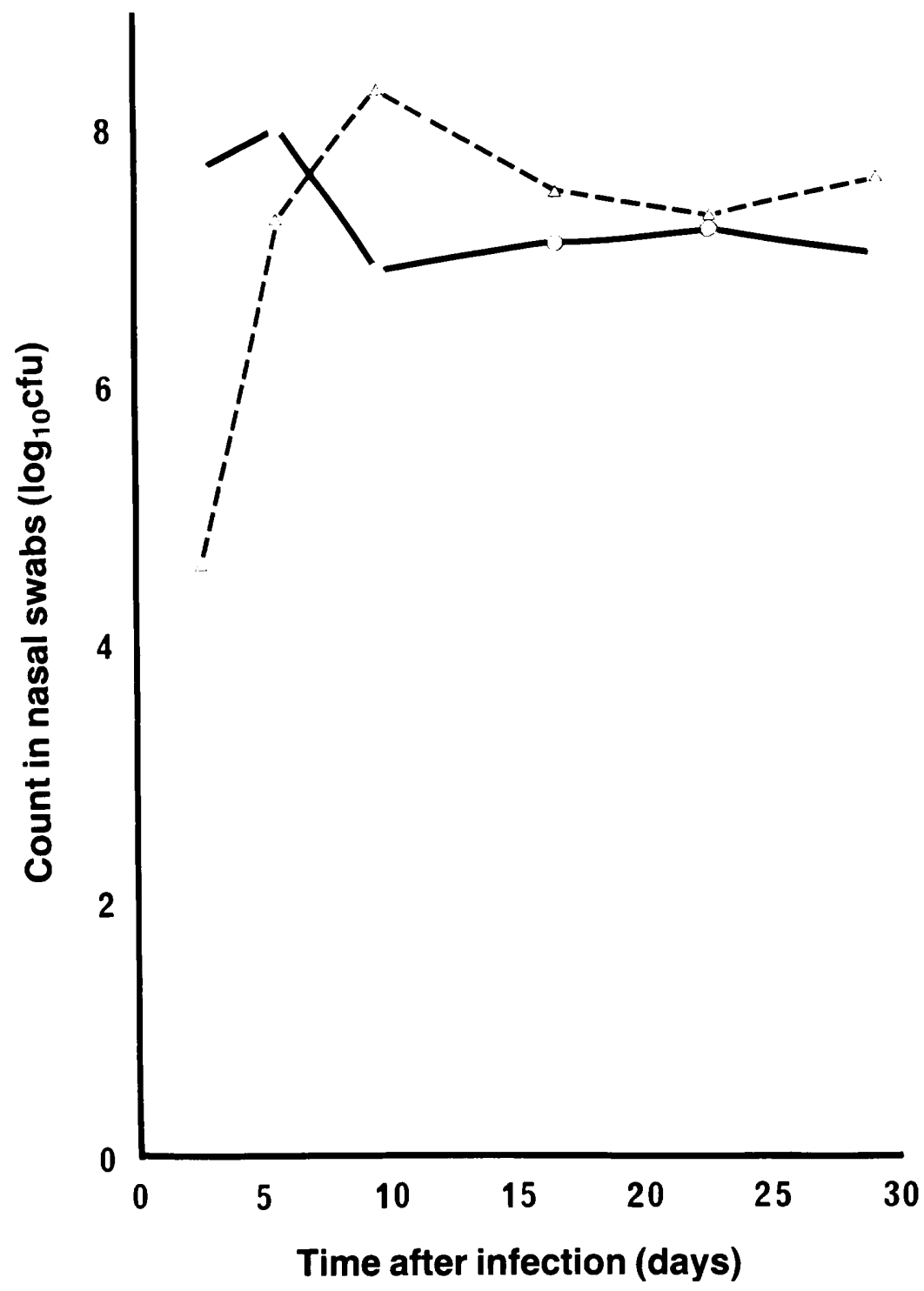

Fig. 1.-Mean nasal counts of three non-AR isolates of B. bronchiseptica (LI, CWT10, CTH19) in gnotobiotic pigs given $9 \cdot 8(O-O)$ or $3 \cdot 0(\Delta---\Delta) \log 10 \mathrm{cfu}$. 
$\left(16.5 \times 10^{3} / \mathrm{mm}^{3} ; \mathrm{p}<0.05\right)$ in those given the lower, but there were no differences between isolates in either litter. All pigs had grade-3 turbinate lesions at 28 days after infection (fig. 2) and there were no differences among isolates. Piglets given $9.8 \log _{10}$ cfu had extensive fibrosing pulmonary lesions mainly in the right anterior lobes. In contrast, seven of 11 piglets given 3.0 $\log _{10} \mathrm{cfu}$ had no macroscopic pneumonia although B. bronchiseptica (mean $5 \cdot 4$ $\log _{10} \mathrm{cfu} / \mathrm{g}$ ) was present in their right cardiac lobes; the other four piglets had mild pulmonary lesions and $B$. bronchiseptica was present (mean $6.5 \log _{10}$ $\mathrm{cfu} / \mathrm{g})$.

\section{Experiment 3: piglets infected with AR isolate 276 for 4, 9 or 24 weeks}

Mean counts in the nasal cavity increased to $7 \cdot 6 \log _{10}$ cfu at 8 days after infection, and declined to $c .4 \cdot 0 \log _{10}$ cfu at 64 days; this count was sustained until 100 days and the two surviving piglets remained intermittently infected until 166 days. The turbinates of the infected piglets were significantly smaller

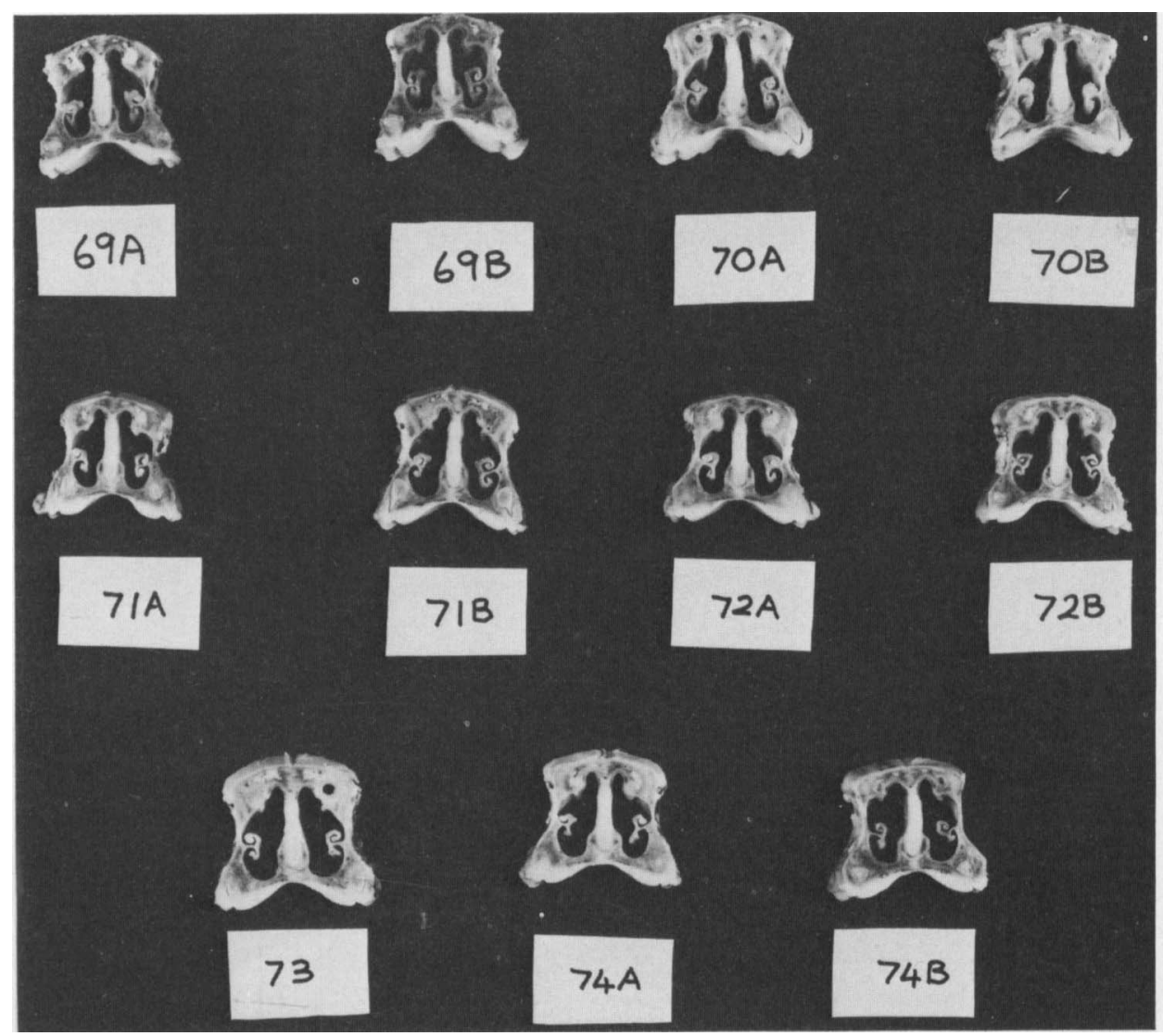

FIG. 2.-Turbinate hypoplasia produced by AR isolates GFS3C(71), AS4(72) and 276(73), and non-AR isolates CWT10(69), LI(70) and CTH19(74). 
than those of the controls ( $p<0.05)$ at 28 and 68 days; however, the turbinates of infected piglets were significantly larger at 68 than at 28 days $(\mathrm{p}<0.01)$, and by 166 days there were no differences between those from infected and control piglets. This indicated that the lesions were not progressive, and that regeneration began after 28 days.

\section{Virulence of B. bronchiseptica in mice}

The mean LD50 of six AR isolates was 7.2 $\log _{10}$ cfu (range 6.6-7.9) and of six non-AR isolates $7 \cdot 0 \log _{10} \mathrm{cfu}$ (range 6.7-7.6). The mean LD50 of phase-III isolates prepared from 276, MH7 and IM5 was 9.9 $\log _{10}$ cfu (range 9.8-10.1). The LD50 dilutions of toxin prepared from AR isolates 276 and $\mathrm{MH} 7$, and non-AR isolate IM5 were 1 in 132, 1 in 82 and 1 in 101 respectively. There were no significant differences in these results except that the phase-III laboratory-derived cultures were $c .600$ times less virulent than the parent phase-I isolates.

\section{DisCUSSION}

The pathogenicity of many bacteria depends on their ability to establish themselves in a susceptible host and to produce toxins or virulence determinants that cause pathological changes and clinical signs of disease. Variations in these properties between strains of $B$. bronchiseptica could, therefore, lead to variations in the clinical manifestations of atrophic rhinitis in pigs. Germ-free piglets were chosen for this study because they made possible the comparison of different isolates in littermates without the occurrence of cross infection, and without interference by passive antibodies and other micro-organisms. Although some piglets died after infection with large numbers of $B$. bronchiseptica, clinical signs in the survivors were mild. Ten isolates - from affected and from AR-free herds - produced turbinate hypoplasia and pneumonia typical of $B$. bronchiseptica infection in gnotobiotic piglets (Brassinne, Dewaele and Gouffaux, 1976); in addition, a slight deviation of the snout was observed occasionally. However, the turbinate and snout lesions were not progressive (Nielsen, Riising and Bille, 1976), even though $B$. bronchiseptica remained in the nasal cavity for 166 days. The pattern of colonisation was similar for all 10 isolates, except that peak bacterial counts occurred a few days later in pigs given smaller doses. The nasal counts in gnotobiotic piglets were similar to those in conventional piglets aged 4-10 weeks from herds with atrophic rhinitis (Rutter, 1981); thus it seems unlikely that our failure to produce progressive lesions could be attributed to inadequate colonisation. Although all the porcine isolates tested produced similar results, subsequent studies have shown that a canine and a human isolate of B. bronchiseptica did not become well established in germ-free piglets, or cause turbinate hypoplasia (Harrison and Rutter, unpublished results); our infection model can, therefore, detect differences between the virulence of different isolates.

Little is known of the pathogenesis of atrophic rhinitis. Guinea-pig 
isolates of $B$. bronchiseptica produced a heat-labile, lethal, dermonecrotic toxin (Evans and Maitland, 1939); extracts from porcine isolates affected beef-heart mitchondria (Harris, Switzer and Harris, 1971); and cell-free extracts of a porcine isolate produced turbinate hypoplasia after repeated instillation into the nasal cavity of pigs (Hanada et al., 1979). On the other hand, invasion of osteoblasts by unidentified bacteria was reported in pigs with atrophic rhinitis (Fetter, Switzer and Capen, 1975). Isolation of B. bronchiseptica from the organs of gnotobiotic piglets suggests that penetration below the mucosa of the nasal cavity may occur, and that toxin production and invasiveness may contribute to the lesions. However, we obtained no evidence for differences in the virulence of 17 isolates that would explain variations in the clinical manifestations of the disease: all the isolates were phase I, they killed gnotobiotic pigs, produced similar turbinate lesions and pneumonia, and stimulated similar antibody and leucocyte responses; there was no effect on weight gain or temperature. There were no morphological or chemical changes in the facial bones of the infected piglets, nor was there any evidence from blood-plasma composition that the organism induced changes in the skeleton as a whole. The effects on bone were confined to the turbinates. Furthermore, in mice the LD50 of live organisms or toxin was similar for isolates from affected and unaffected herds, although three laboratory-derived phase-III cultures were less virulent than the parent strains. We conclude, therefore, that there were no significant differences in virulence between these porcine isolates of $B$. bronchiseptica.

Our failure to detect such differences contrasts with the results of Miniats and Johnson (1980) and Skelly et al. (1980), who found that some porcine isolates did not produce turbinate lesions. Colonisation studies were not reported by these authors and it is not clear whether the differences were associated with establishment or virulence; they might also be attributable to the use of older conventional pigs (Skelly et al., 1980), or to the delay (6-8 weeks) in killing them. In future studies it would be helpful to kill pigs 3-4 and 8-9 weeks after infection to detect early and persistent turbinate changes.

The discrepancy between our results and those of Martineau et al. (1980), who produced turbinate changes in gnotobiotic pigs only by infecting them with more than $5.5 \log _{10} \mathrm{cfu}$, is difficult to explain. We produced heavy colonisation of the nasal cavity consistently with a single dose $\left(3.0 \log _{10} \mathrm{cfu}\right)$ of many isolates of $B$. bronchiseptica, and in one pig colonisation and turbinate lesions were produced with $c$. 65 organisms of AR-isolate 276 (Rutter, unpublished results).

Although we were unable to reproduce the progressive lesions of atrophic rhinitis with $B$. bronchiseptica in gnotobiotic piglets, the production of turbinate hypoplasia with isolates from unaffected herds explains the presence of minor turbinate abnormalities in these herds. It would be helpful to designate such herds as being affected with turbinate hypoplasia associated with bordetellosis to differentiate them from herds with atrophic rhinitis. To explain the aetiology of atrophic rhinitis, other factors are needed and three hypotheses could be advanced: (1) the pathogenicity of $B$. bronchiseptica might be enhanced by the presence of other micro-organisms; (2) B. bronchiseptica 
might enhance the pathogenicity of other micro-organisms; (3) other microorganisms might cause atrophic rhinitis, bordetellosis being a concomitant but separate infection. More investigations are needed to determine which of these hypotheses is correct.

We are indebted to Dr I. M. Smith for isolate 276, and to Mr D. C. Davies, Dr D. Baggott, and $\mathrm{Mr}$ M. J. Dennis and his staff for providing and rearing the gnotobiotic piglets. The assistance of Miss N. Rolley, Miss D. Allday, Mr C. Mallinson, and Mr P. Gleed is gratefully acknowledged.

\section{REFERENCES}

Alexander, T. J. L., Thornton, K., Boon, G., Gush, A.F. And Lysons, R. J. 1980. Medicated early weaning to obtain pigs free from pathogens endemic in the herd of origin. Vet. Rec., 106, 114.

Brassinne, M., Dewaele, A. and Gouffaux, M. 1976. Intranasal infection with Bordetella bronchiseptica in gnotobiotic piglets. Res. vet. Sci., 20, 162.

Cameron, R. D. A., Giles, C. J. ANd Smith, I. M. 1980. The prevalence of Bordetella bronchiseptica and turbinate (conchal) atrophy in English pig herds in 1978-79. Vet. Rec., $107,146$.

Done, J. T., Richardson, M. D. and Hebert, C. N. 1964. Ministry of Agriculture, Fisheries and Food. Animal disease surveys, report No. 3, London, HMSO, p. 31.

Duncan, J. R., Ramsey, F. K. AND Switzer, W. P. 1966. Pathology of experimental Bordetella bronchiseptica infection in swine: pneumonia. Am. J. vet. Res., 27, 467.

Duncan, J. R., Ross, R. F., Switzer, W. P. and Ramsey, F. K. 1966. Pathology of experimental Bordetella bronchiseptica infection in swine: atrophic rhinitis. Am. J. vet. Res., $27,457$.

Evans, D. G. AND Maitland, H. B. 1939. The toxin of Br. bronchiseptica and the relationship of this organism to H. pertussis. J. Path. Bact., 48, 67.

Fetter, A. W., Switzer, W. P. AND CAPEN, C. C. 1975. Electron microscopic evaluation of bone cells in pigs with experimentally induced Bordetella rhinitis (turbinate osteoporosis). Am. J. vet. Res., 36, 15.

Giles, C. J., Smith, I. M., Basker ville, A. J. ANd Brothwell, E. 1980. Clinical bacteriological and epidemiological observations on infectious atrophic rhinitis of pigs in southern England. Vet. Rec., 106, 25.

Hanada, M., Shimoda, K., Tomita, S., Nakase, Y. and Nishiyama, Y. 1979. Production of lesions similar to naturally occurring swine atrophic rhinitis by cell-free sonicated extract of B. bronchiseptica. Jap. J. vet. Sci., 41, 1.

Harris, D. L., Switzer, W. P. AND Harris, R. A. 1971. A suggested mechanism for the pathogenesis of infectious atrophic rhinitis. Can. J. comp. Med., 35, 318.

JeNKINS, E. M. 1978. An agglutination test for the detection of Bordetella bronchiseptica infection in swine. Can J. comp. Med., 42, 286.

Martineau, G., Josse, M., Martineau-Doize, B. and Colgnoul, F. 1980. Atrophic rhinitis caused by Bordetella bronchiseptica: determination of the minimum infectious dose (MID) on gnotobiotic piglets. In Proceedings of the 6th congress of the International Pig Veterinary Society held in Copenhagen, P201.

MEYER, R. C. AND BEAMER, P. D. 1973. Bordetella bronchiseptica infections in germ-free swine: an experimental pneumonia. Vet. Path., 10, 550.

Miniats, O. P. AND Johnson, J. A. 1980. Experimental atrophic rhinitis in gnotobiotic pigs. Can. J. comp. Med., 44, 358.

NAKASE, Y. 1957a. Studies on Haemophilus bronchisepticus. I. The antigenic structures of $H$. bronchisepticus from guinea pig. Kitasato Arch. exp. Med., 30, 57.

NakaSE, Y. 1957b. Studies on Haemophilus bronchisepticus. II. Phase variation of $H$. bronchisepticus. Kitasato Archs exp. Med., 30, 73.

Nielsen, N. C., RiIsing, H.-J. AND Bille, N. 1976. Experimental reproduction of atrophic rhinitis in pigs reared to slaughter weight. In Proceedings of the 4th congress of the International Pig Veterinary Society, held in Ames, Iowa, P1. 
REED, L. J. AND MUench, H. 1938. A simple method of estimating fifty per cent end points. Am. J. Hyg., 27, 493.

Robinson, R., Roughan, M. E. And Wagstaff, D. F. 1971. Measuring inorganic phosphate without using a reducing agent. Ann. clin. Biochem., 8, 168.

Ross, R. F., Switzer, W. P. AND DunCan, J. R. 1967. Comparison of pathogenicity of various isolates of Bordetella bronchiseptica in young pigs. Can. J. comp. Med., 31, 53.

RUTTER, J. M. 1981. Quantitative observations on Bordetella bronchiseptica infection in atrophic rhinitis of pigs. Vet. Rec., 108, 451.

Skelly, B. J., Pruss, M., Pellegrino, R., Andersen, D. And Abruzzo, G. 1980. Variation in degree of atrophic rhinitis with field isolates of Bordetella bronchiseptica. In Proceedings of the 6th congress of the International Pig Veterinary Society, held in Copenhagen, P210.

Steel, R. G. D. AND TORRIE, J. H. 1960. Principles and procedures of statistics, McGraw-Hill, London, p. 242.

Switzer, W. P. AND Farrington, D. O. 1976. Infectious atrophic rhinitis. In Diseases of swine, edited by H. W. Dunne and A. D. Leman, 4th ed., Iowa State University Press, Ames, Iowa, p. 687.

Tavernor, W. D., Trexler, P. C., Vaughan, L. C., Cox, J. E. and Jones, D. G. C. 1971. The production of gnotobiotic piglets and calves by hysterotomy under general anaesthesia. Vet. Rec., 88, 10.

TreXLeR, P. C. 1971. Microbiological isolation of large animals. Vet. Rec., 88, 15. 